\title{
PLAN ANUAL Y CONTROL DE UTILIDADES COMO ESTRATEGIA DE INTEGRACIÓN DE LAS MYPES INDUSTRIALES PERUANAS
}

\author{
ANNUAL PLAN AND CONTROL OF EARNINGS AS INTEGRATION \\ STRATEGY OF THE PERUVIAN INDUSTRIAL PYMES
}

\author{
Raúl A. Arrarte Mera* \\ Docente Principal de la Facultad de Ciencias Contables, UNMSM
}

[Recepción: Setiembre de 2010 / Conformidad: Octubre de 2010]

\section{RESUMEN}

En el Perú, según estudios realizados por el Ministerio de Trabajo y Promoción Social, el 95\% de las empresas esta representado por Micro y Pequeñas Empresas (MYPES) y sólo el 5\% de ellas personifica a la Gran Empresa. Asimismo, publicaciones de la Superintendencia de Administración Tributaria (SUNAT) expresan que el $60 \%$ de las empresas nacionales son informales. El trabajo que exponemos a continuación, está referido al área de CONTABILIDAD - INFORMACIÓN PARA LA TOMA DE DECISIONES (Contabilidad de Costos: Modelos y Enfoques), busca los siguientes objetivos:

1. Permitir una mayor comprensión de la importancia que tiene, conocer por adelantado, los costos de producción u operación y los resultados de cada empresa del sector industrial preferentemente exportador.

2. Relevar la jerarquía que estas técnicas tienen para el ejercicio de una sana competencia. Impulsando a la administración a planificar el empleo más económico de la mano de obra, de los materiales y del capital.

3. Desarrollar una cultura prospectiva en el recurso humano empresarial medio, inculcando en todos los niveles de la administración, el hábito de la consideración oportuna, cuidadosa y apropiada de todos los factores pertinentes, antes de tomar decisiones de trascendencia.

\begin{abstract}
The present research aims to demonstrate the need for mandatory use of the technical basis and implementation of a uniform system of Planning and Control Master Profit or short-term budget for all Small and Medium Enterprises (SMEs) based in the industrial PERU, prior to the use of Strategic Business Plan projected the long term.

In our country, according to studies by the Ministry of Labour and Social, 95\% is represented by Small and Medium Enterprises (SMEs) and only 5\% personifies the large corporations. Also, publications of the Superintendency of Tax Administration (SUNAT) state that $60 \%$ of domestic enterprises are informal.

In the global market the domestic firm, has to deal with large organizations, a very intense competitive situation and hostile, that they enjoy using the Planning and Utility Control on a daily basis, but the average entrepreneur unknown. Noteworthy among the benefits to the micro level, encouraging healthy competitionandimprovingbusinessefficiency at the national and international levels, producing also the employer strengthening the regional integration processes such as the Andean Community of Nations (CAN). The targets we have set to develop this work are:

1. Allow greater understanding of the importance, to know in advance, costs of production or operation and
\end{abstract}

* Contador Público Colegiado, Doctor en Ciencias Contables y Empresariales y Magíster en Admnistración. Actual Director del Instituto de Investigaciones en Ciencias Financieras y Contables. E-mail: raularrarte@speedy.comp.pe 
La meta específica es proponer un Modelo de Plan Anual y Control de Utilidades de Corto Plazo para las MYPES Industriales Peruanas. La metodología utilizada para el desarrollo de este trabajo de investigación es de tipo Inductivo y Deductivo, aplicado con el propósito de establecer las conclusiones y generalizar los resultados. De Síntesis, para establecer la relación causa-efecto entre los objetos que componen los elementos de la investigación. Y la técnica de Análisis Documental, que nos permitirá obtener la información principalmente de textos, que consiste en consultar la bibliografía integrada por libros de costos y gestión de costos referidos al Plan Anual de Utilidades o Plan Maestro.

El resultado de este trabajo pretende demostrar la necesidad del uso obligatorio de los fundamentos técnicos e implementación de un sistema uniforme de Plan Anual y Control de las Utilidades a corto plazo, como estrategia de integración, para todas las Micro y Pequeñas Empresas (MYPES) industriales radicadas en el PERÚ, como paso previo al empleo del Plan Estratégico del Negocio proyectado al largo plazo. Con lo que se lograría recomendar el establecimiento de $\mathrm{Pla}$ nes Curriculares específicos en nuestra $\mathrm{Fa}$ cultad de Ciencias Contables para preparar especialistas en Sistemas de Planeamiento y Control de Utilidades tanto para las MYPES como para las distintas ramas de la actividad económica, en coordinación con la Contaduría Pública de la Nación y la Federación de Contadores Públicos del Perú, contribuyendo con ello a reducir la informalidad de las actividades empresariales, mejorar la toma de decisiones, optimizar el uso de los escasos recursos de las MYPES, apoyando a los fines de nuestra Universidad y al desarrollo económico y social del país.

Palabras clave: Plan anual de utilidades. Plan maestro, planeamiento y Control de utilidades, Presupuesto anual.

\section{INTRODUCCIÓN}

En el Perú, según estudios realizados por el Ministerio de Trabajo y Promoción Social, el 95\% está representado por Micro y Pequeñas Empresas (MYPES) y sólo el 5\% personifica a la Gran Empresa. Asimismo, publicaciones performance of each company in the industrial sector mainly exporter.

2. Relieve the hierarchy that these techniques have for the exercise of healthy competition. Urging the administration to plan more economical use of labor, materials and capital.

3. Develop a foresight culture in human resources business environment, instilling in all levels of government, the habit of proper consideration, careful and appropriate use of all relevant factors before making major decisions.

With a specific goal: To propose a model Annual Plan and Utility Control for ShortTerm Industrial Peruvian SMEs.

With what could be achieved to recommend the establishment of specific curricula in our School of Accounting to prepare specialists in Planning and Control Systems of Utilities both for SMEs and for the different branches of economic activity thus helping to reduce informality business operations, improve decision making, optimize the use of scarce resources of SMEs, supporting the goals of our University and to the economic and social development.

Key words: Annual plan and control of earnings, integration strategy, the Peruvian industrial PYMES.

de la Superintendencia de Administración Tributaria (SUNAT) expresan que el 60\% de las empresas nacionales son informales. Además, en el mercado global la empresa nacional, tiene que enfrentarse a las grandes organizaciones, a una situación competitiva 
muy intensa y hostil, que precisamente utilizan el Planeamiento y Control de Utilidades en forma cotidiana, pero que el empresario medio desconoce. Destaca, entre los beneficios a obtener a nivel microeconómico, el fomento de una competencia sana y el mejoramiento de la eficiencia empresarial en el orden nacional e internacional, produciendo además el fortalecimiento empresario al mejorar la productividad de las empresas y la calidad de vida de millones de trabajadores.

\section{EL OBJETIVO GENERAL}

Proponer un Modelo de Plan Anual y Control de Utilidades de Corto Plazo, para las PYMES (Pequeñas y Medianas Empresas), del sector industrial peruano.

\section{METAS ESPECÍFICAS}

a) Permitir una mayor comprensión de la importancia que tiene conocer, por adelantado, los costos de producción u operación y los resultados de cada empresa del sector industrial preferentemente exportador.

b) Relevar la importancia que estas técnicas tienen para el ejercicio de una sana competencia. Impulsando a la administración a planificar el empleo más económico de la mano de obra, de los materiales y del capital.

c) Desarrollar una cultura prospectiva en el recurso humano empresarial medio, inculcando en todos los niveles de la administración, el hábito de la consideración oportuna, cuidadosa y apropiada de todos los factores pertinentes, antes de tomar decisiones de trascendencia.

\section{CONTEXTO DE LA PROBLEMÁTICA ABORDADA}

En nuestro país, según estudios realizados por el Ministerio de Trabajo y Promoción Social, el 95\% está representado por Pequeñas y Medianas Empresas (PYMES) y sólo el $5 \%$ personifica a la Gran Empresa. Asimismo, publicaciones de la Superintendencia de Administración Tributaria (SUNAT) expresan que el $60 \%$ de las empresas nacionales son informales. La enseñanza de un Plan Anual y Planificación de Utilidades podría ser una herramienta que ayudaría a disminuir la informalidad empresarial.

\section{ENFOQUE}

En el mercado global, la empresa nacional tiene que enfrentarse a las grandes organizaciones, a una situación competitiva muy intensa y hostil, que precisamente utilizan el Planeamiento y Control de Utilidades en forma cotidiana, pero que el empresario medio desconoce. Destaca, entre los beneficios a obtener a nivel microeconómico, el fomento de una competencia sana y el mejoramiento de la eficiencia empresarial en el orden nacional e internacional, produciendo además el fortalecimiento empresario en los procesos de integración regional, como por ejemplo la Comunidad Andina de Naciones (CAN). 


\section{INFORMACIÓN ACTUALIZADA Y DIRECTAMENTE RELACIONADA AL PROBLEMA}

\begin{tabular}{|l|}
\begin{tabular}{|l|c|c|c|}
\hline \multicolumn{1}{|c|}{ Pector Económico } & $\begin{array}{c}\text { Participación } \\
\text { Sectorial } / 1\end{array}$ & $\begin{array}{c}\text { MYPE en cada } \\
\text { sector } / 2\end{array}$ & $\begin{array}{c}\text { Participación de } \\
\text { MYPE } / 3\end{array}$ \\
\hline Agricultura & 11.6 & 90.0 & 10.4 \\
\hline Pesca & 1.0 & 30.0 & 0.3 \\
\hline Minería & 2.5 & 5.0 & 0.1 \\
\hline Manufactura & 23.1 & 30.0 & 6.9 \\
\hline Construcción & 8.8 & 35.0 & 3.1 \\
\hline Servicios & 53.0 & 40.0 & 21.2 \\
\hline Total & 100.0 & & 42.1 \\
\hline
\end{tabular}
\end{tabular}

1/ Contribución de cada sector en el PBI. Fuente INEI

Fuente: Ministerio de Trabajo y Promoción Social. Datos 2007, 2008 y 2009.

\section{Composicion MYPE segun formalidad tributaria}

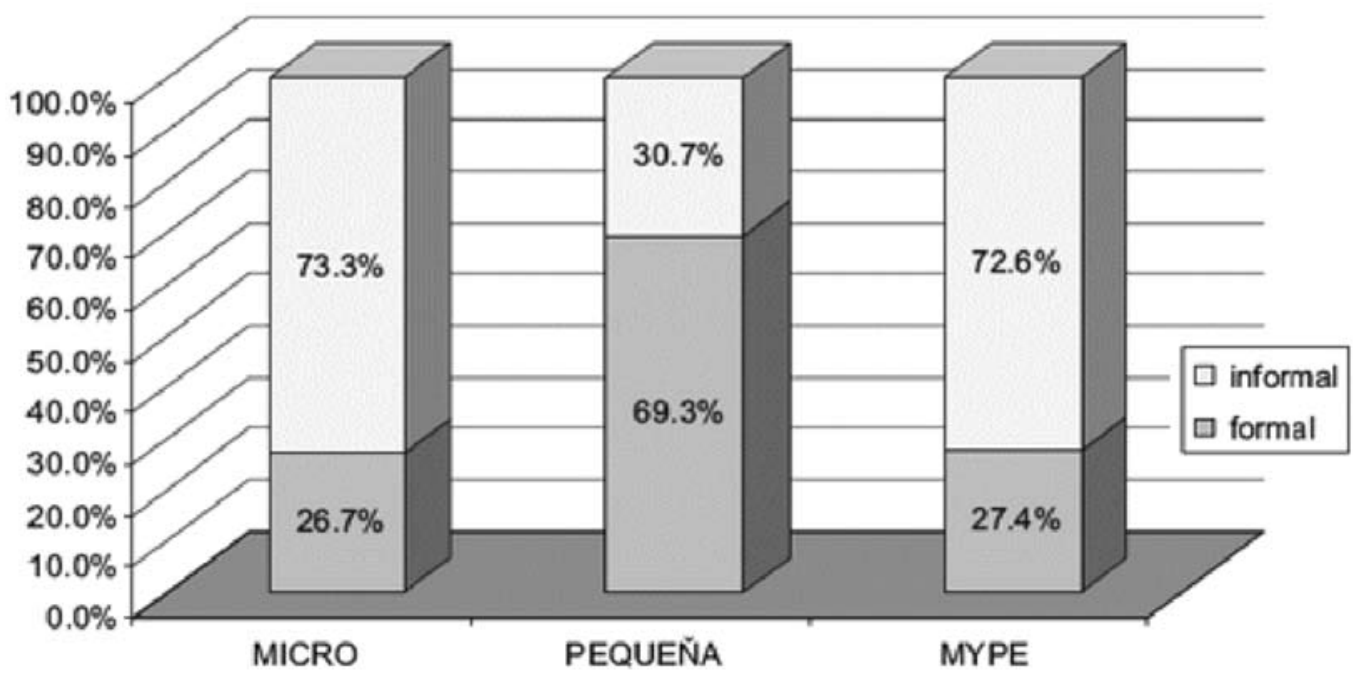

Fuente: Ministerio de Trabajo y Promoción Social. Datos 2007, 2008 y 2009 por sectores Micro, Pequeña - MYPE

La principal fuente de las empresas para obtener ventajas competitivas es el conocimiento. Cuando de la noche a la mañana cambian los mercados, proliferan las tecnologías, se multiplican los competidores y los productos quedan obsoletos, sólo para que las empresas alcancen el éxito de una forma consistente deben crear nuevo conocimiento, gestionar el que poseen, difundirlo a través de toda la empresa e incorporarlo como un nexo tecnológico a sus procesos, productos y servicios.

Una de las herramientas para lograr alta competitividad industrial en el Perú, es mediantelacreacióndemodelosdegestióndel 
conocimiento, tanto para el sector industrial como para universidades, impulsando así la investigación y desarrollo (I\&D) en el país. El tema que estamos planteando es un modelo de gestión del conocimiento referido al Plan Anual y Control de Utilidades, exigiendo que su cumplimiento sea de carácter obligatorio, esperando obtener resultados positivos en productividad y perspectivas de desarrollo en un mundo donde las Normas Internacionales de Contabilidad (NIC's), y las Normas Internacionales de Información Financieras (NIIF's), pretenden normarlo todo.

Destacamos que el documento en extenso de esta ponencia, es un texto de consulta complementaria para los estudiantes de la asignatura de Costos y Prespuestos que se dicta en la Facultad de Ciencias Contables para optar el Titulo Profesional de Contador Público.

\section{MARCO CONCEPTUAL PARA LA PREPARACIÓN DEL PLAN ANUAL DE UTILIDADES}

\section{Ventajas y limitaciones de los presupuestos}

\section{Ventajas}

1. Obliga a la planeación y a la ejecución de planes.

2. Proporciona los criterios para la evaluación del desempeño.

3. Facilita la coordinación de actividades y fomenta la comunicación

4. Apoya en la detección de problemas internos.

5. Obliga a considerar oportunamente las políticas básicas.

6. Exige una adecuada y sana estructura de organización; esto es, debe haber una clara asignación de responsabilidades para cada función en la empresa.

7. Impulsa a todos los miembros de la administración, desde arriba hasta abajo, a participar en el establecimiento de metas y planes.

8. Exige a los gerentes departamentales hacer planes en armonía con los planes de otros departamentos y con los de la empresa en su conjunto.

9. Obliga a que la Gerencia General consigne en cifras lo que considera necesario para un desempeño satisfactorio.

10. Exige la disponibilidad de datos contables históricos suficientes y apropiados.

11. Impulsa a la administración a planificar el empleo más económico de factores de la producción.

12. Inculca, en todos los niveles de la empresa, el hábito de la consideración oportuna, cuidadosa y apropiada de todos los factores pertinentes antes de tomar decisiones de trascendencia.

13. Reduce el costo al ampliar el espacio del control, pues se requieren menos supervisores.

14. Libera a los ejecutivos de innumerables problemas cotidianos internos mediante políticas predeterminadas y relaciones bien definidas de autoridad. Facilitándoles así, más tiempo para la planificación y reflexión creativas.

15. Tiende a eliminar la nube de incertidumbre que existe en muchas organizaciones, especialmente entre los niveles inferiores de la administración, relativa a las políticas básicas y los objetivos de la empresa.

16. Señala con precisión la eficiencia y la ineficiencia y hacer un autoanalisis de la compañía.

17. Promueve la comprensión, entre los miembros de la administración, de los problemas de sus compañeros de trabajo.

18. Obliga a la administración a prestar debida atención al efecto de las condiciones generales de los negocios.

19. Ayuda a conseguir crédito bancario; los bancos generalmente exigen una proyec- 
ción de las futuras operaciones y de los flujos de efectivo que apoyen a los préstamos solicitados.

20. Verifica el avance o la ausencia del mismo, hacia los objetivos de la empresa.

21. Obliga al reconocimiento y a la acción correctiva (incluyendo las recompensas).

22. Premia el desempeño sobresaliente y busca corregir el desempeño desfavorable.

23. Mueve a la Gerencia General a considerar las futuras tendencias y condiciones esperadas.

24. Hay que prever antes que solucionar los problemas.

\section{Limitaciones}

1. Está basado en estimaciones.
2. Debe ser adaptado constantemente a los cambios de importancia.

3. Su ejecución no es automática.

4. Es un instrumento que no debe sustituir a la administración.

5. No se deben esperar resultados demasiado pronto.

6. Resta flexibilidad a la administración del negocio.

7. Crea toda clase de problemas de comportamiento.

8. Impone una camisa de fuerza a la administración.

9. Añade un nivel de complejidad que no es necesario.

10. Es demasiado costoso, aparte del tiempo que invierte la administración.

\section{Proceso de planificación y control de las utilidades}

\begin{tabular}{|c|c|c|}
\hline $\begin{array}{l}\text { FUNCIÓN DE LA } \\
\text { ADMINISTRACIÓN }\end{array}$ & $\begin{array}{l}\text { FASES SECUENCIALES DEL PROCESO DE PLANIFICACIÓN Y CONTROL } \\
\text { DE UTILIDADES. }\end{array}$ & $\begin{array}{l}\text { RESPONSABILIDAD } \\
\text { PRIMARIA }\end{array}$ \\
\hline \multirow{8}{*}{ Planear } & 1. Variables Externas pertinentes. Identificar y evaluar. & \multirow{5}{*}{$\begin{array}{l}\text { Administración } \\
\text { Ejecutiva }\end{array}$} \\
\hline & 2. Objetivos generales del negocio. Desarrollar o modificar. & \\
\hline & 3. Metas específicas de la empresa. Desarrollar en congruencia con punto 2 . & \\
\hline & $\begin{array}{l}\text { 4. Estrategias de la empresa. Especificar los principales impulsos para alcanzar los } \\
\text { objetivos y las metas. }\end{array}$ & \\
\hline & $\begin{array}{l}\text { 5. Instrucciones de la administración ejecutiva para la planificación. Especificar linea- } \\
\text { mientos de la planificación para los gerentes en base a los puntos 1-4 anteriores. }\end{array}$ & \\
\hline & 6. Planes de proyectos. Desarrollar y evaluar cada proyecto. & \multirow{3}{*}{$\begin{array}{l}\text { Administración de } \\
\text { Nivel Medio }\end{array}$} \\
\hline & 7. Plan estratégico de largo plazo de utilidades. Para 3 a 5 años. & \\
\hline & 8. Plan táctico corto plazo de utilidades. Desarrollar para el año entrante. & \\
\hline Dirigir & 9. Estructuración de planes de utilidades. Ejecutar a lo largo del año presupuestal. & \multirow{3}{*}{$\begin{array}{l}\text { Todos los niveles de la } \\
\text { Administración }\end{array}$} \\
\hline \multirow{2}{*}{ Controlar } & 10. Informes de desempeño. Preparar informes mensuales por áreas de responsabilidad. & \\
\hline & 11. Seguimiento. Proveer retroalimentación, emprender acción correctiva y replanificar. & \\
\hline
\end{tabular}

Fuente: Glenn Welsch. Planificación y Control de Utilidades, p. 78. 


\section{ESTRUCTURA DEL PLAN ANUAL DE UTILIDADES}

El Presupuesto General o Maestro está conformado por presupuestos parciales. Para Charles Horngren ${ }^{1}$, un presupuesto Maestro es en esencia, un análisis más extenso del primer año de un plan a largo plazo. Un presupuesto es una expresión formal, cuantitativa de los planes de la administración. Un presupuesto maestro resume las actividades planeadas de todas las subunidades de una organización: ventas, producción, distribución y finanzas. Por tanto, el presupuesto maestro es un plan de negocios periódico que incluye un conjunto coordinado de programas de operación y estados financieros detallados. Este incluye proyecciones de ventas, gastos, ingresos y desembolsos de efectivo y balances generales. Algunas veces los presupuestos maestros también se llaman Estados Proforma, un sinónimo de estados financieros proyectados.

Los componentes de un presupuesto varían de una organización a otra, pero los elementos comunes principales son los siguientes:

- Presupuesto de operaciones. Que comprende todos los ingresos, costos y gastos, se enfoca en el estado de resultados y sus programas de apoyo.

- Presupuesto de inversiones. Se orienta a las decisiones de inversión de corto y largo plazo y fundamentalmente a la presupuestación de capital que tendrán un impacto sobre la futura posición en el mercado de la firma en sus actuales líneas de productos.

- Presupuesto financiero. Se centra en los efectos que el presupuesto de operación y el de inversiones tendrán sobre el efectivo.

\section{El presupuesto de operaciones}

Se enfoca en los estados de resultados y sus programas de apoyo como es el caso del punto de equilibrio. Son estimados que en forma directa el proceso tiene que ver con la parte neurológica de la Empresa, desde la producción misma hasta los gastos que conlleva ofertar el producto terminado. Son componentes de este rubro:

- Presupuesto de venta.

- Presupuesto de producción.

- Presupuesto de requerimiento de materiales directos.

- Presupuesto mano de obra directa.

- Presupuesto de costos indirectos de fabricación.

- Presupuesto costo de artículos vendidos.

- Presupuesto gastos de ventas.

- Presupuesto gastos de administración.

- Presupuesto de gastos financieros.

- Estado de ganancias y pérdidas proyectado.

\section{El presupuesto de ventas}

Son estimados que tienen como prioridad establecer el nivel de ventas real y proyectado de una empresa, para determinado límite de tiempo. Que para nuestro caso está referido a un año calendario equivalente a doce meses. La elaboración de un Presupuesto de Venta, constituye el problema primario $y$ fundamental que es necesario resolver satisfactoriamente para una buena elaboración presupuestaria. Es clave definir la tendencia futura: estacionaria, creciente o decreciente.

\section{Información necesaria}

- Línea de productos que comercializa la empresa.

1 Horngren, Charles T. Sandem, Gary L. "Contabilidad Administrativa”. Novena edición. Editorial Prentice-Hall Hispanoamericana S.A., México, 1994, p. 248. 
- El valor de venta unitario de cada producto.

- El nivel de venta de cada producto.

A los efectos de ejemplificar nuestra exposición, desarrollaremos el ejercicio de un proceso integral de planeamiento presupuestal. A este fin asumimos el hipotético caso de una empresa industrial, del Sector Textil, con producción por procesos continuos. Una empresa que elabora y comercializa cinco productos de consumo masivo (A - B - C D - E).

\section{Objetivos de la compañía}

1. Seguir suministrando productos de primera calidad a nuestros clientes.

2. Adoptar un método administrativo progresista y brindar una motivación positiva al personal de la organización.

3. Instalar un sencillo pero efectivo sistema de contabilidad.

\section{Metas específicas}

1. Percibir una utilidad bruta de por lo menos el $25 \%$ sobre las ventas netas.

2. Aumentar el ingreso por ventas a una tasa anual del $10 \%$.

3. Generar suficiente efectivo cada año para atender al normal desarrollo de las actividades empresariales.

4. Renovar las instalaciones para mejorar la eficiencia y hacerla más cómoda para los trabajadores.

Los parámetros que se deben definir antes de proceder al cálculo presupuestal, son los siguientes: nivel de inflación anual, tasa de devaluación anual, el costo del dinero (Tasa Anual en Moneda Nacional TAMN, Tasa Anual en Moneda Extranjera TAMEX),
Tasa Tributaria del Impuesto a la Renta y la Unidad Impositiva Tributaria (UIT). Después de un análisis y apreciación de las condiciones internas y externas: el producto, el valor de venta, los canales de distribución, la fuerza de ventas, la publicidad y promoción. El mercado, el ambiente económico, político, social, y el ambiente legal, se llegan finalmente a definir la distribución mensual de las ventas.

\section{El presupuesto de producción}

El tercer paso es programar esta producción por subperiodos ${ }^{2}$.

\section{Información necesaria}

- Presupuesto de ventas por línea de productos terminados en unidades.

- Inventario final de productos terminados en unidades.

- Inventario inicial de productos terminados en unidades.

\section{Fórmula del presupuesto de producción en unidades $=$}

Presupuesto de ventas + Inventario final deseado - Inventario inicial

Según Welsch, tres objetivos se persiguen en la planificación de la producción:

1. Proveer suficientes artículos para cumplir las necesidades de venta de los subperiodos.

2. Mantener los niveles del inventario dentro las limitaciones que fijan las políticas.

3. Fabricar los artículos tan económicamente como sea posible.

2 Welsch, Glenn; Hilton, Ronald W; Gordon, Paul N. "Presupuestos. Planificación y Control de Utilidades”. Editorial Prentice Hall Hispanoamericana S A. Quinto Edición. Página 227. Año 1990. México. 


\section{Presupuesto de requerimiento de materiales directos}

En este aparte tenemos:

1. El Presupuesto de Consumo de Materiales Directos.

2. El Presupuesto de Compras de Materiales Directos.

\section{Presupuesto de consumo de materiales directos}

Aproximadamente, al mismo tiempo que se prepara el presupuesto de compras y se piden los materiales directos requeridos, es necesario preparar el Presupuesto de Consumo de Materiales Directos.

\section{Información necesaria}

- Presupuesto de la producción planeada en unidades.

- Tasa de uso de la materia prima por kilo, libra, tonelada, etc.

- Valor de consumo por unidades.

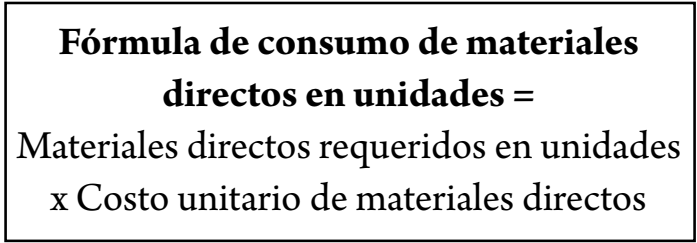

El método desarrollado por la empresa para el control de inventarios se denomina Compras Justo a Tiempo. Su objetivo principal es minimizar los niveles del inventario y los consiguientes costos. Significa que los materiales no se compran sino hasta el momento en que se necesitan para la producción.

\section{El presupuesto de compras de materiales directos}

Son estimados de compras preparado bajo condiciones normales de producción, mientras no se produzca una carencia de materiales esto permite que la cantidad se pueda fijar sobre un estándar determinado para cada tipo de producto así como la cantidad presupuestada por cada línea, debe responder a los requerimiento de producción, el departamento de compras debe preparar el programa que concuerde con el presupuesto de producción, si hubiere necesidad de un mayor requerimiento se tomara la flexibilidad del primer presupuesto para una ampliación oportuna y así cubrir los requerimiento de producción.

Con base en esta hoja, el Departamento de Compras prepara los programas de compras y entrega, que deben estar estrechamente coordinados con el presupuesto de producción y con los programas de entrega del proveedor. El presupuesto de suministros y materiales indirectos se incluye en el presupuesto de costos indirectos de fabricación ${ }^{3}$.

\section{Materia prima}

Es el principal recurso que se usa en la producción; este se transforma en bienes terminados con la ayuda de la mano de obra y los costos indirectos de fabricación.

- Directos: Son todos aquellos que pueden identificarse en la fabricación de un producto terminado, fácilmente se asocian con éste y representan el principal costo de materiales en la elaboración de un producto.

3 Polimeni, Ralph S; Fabozzi, Frank J; Adelberg, Arthur H. Contabilidad de Costos. Conceptos y Aplicaciones para la Toma de Decisiones Gerenciales. Tercera edición. Santa Fe de Bogotá: Editorial McGraw-Hill, 1994; pp. 350 y 351. 
- Indirectos: Son los que están involucrados en la elaboración de un producto, pero tienen una relevancia relativa frente a los directos.

\section{Información necesaria}

- Presupuesto consumos de materias primas y suministros en unidades.

- Inventario final de materiales en unidades.

- Inventario inicial de materiales en unidades

- Valor de compra de los materiales por unidad.

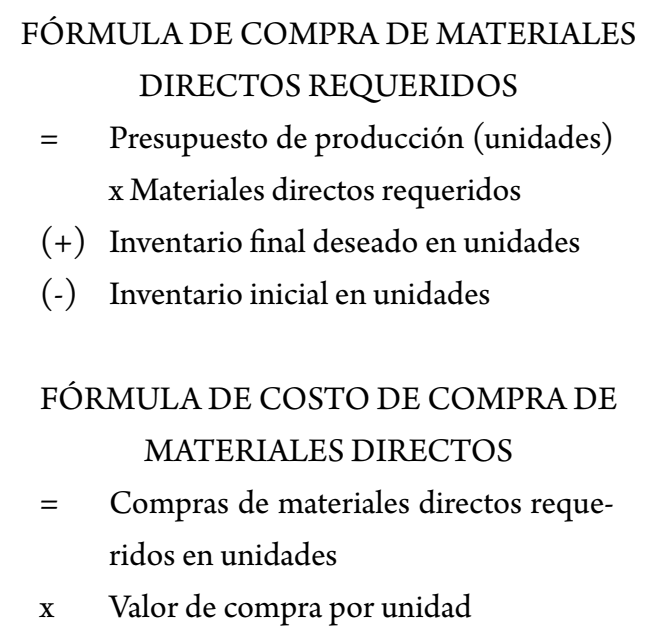

Como la empresa utiliza el método Compras Justo a Tiempo, no existen inventarios de seguridad. Por tanto el costo de los consumos es igual al Costo de las Compras. La política de adquisiciones es $60 \%$ al contado y $40 \%$ al crédito, pagadero a 30 días de las compras.

\section{Presupuesto de mano de obra directa}

Es el diagnóstico requerido para contar con una diversidad de factor humano capaz de satisfacer los requerimientos de producción planeada.

Si el presupuesto para el año entrante requiere menos trabajadores, deben ser suspendidos después de tener en cuenta las habilidades y los derechos de antigüedad de cada uno de ellos, de acuerdo con la política de la compañía o el convenio colectivo entre el Sindicato y la Empresa ${ }^{4}$.

Las ventajas de lograr niveles estables en la producción, permiten:

1. La estabilidad en el empleo que a su vez, facilita:

a) Una mejoría en la moral y autoestima personal $y$, por consiguiente, una mayor eficiencia en el desempeño de los trabajadores.

b) Una menor rotación del personal.

c) Atracción de mejores empleados hacia la empresa.

d) Reducción en los gastos de capacitación de los nuevos empleados.

2. Economía en la compra de materias primas y componentes, como consecuencia de:

a) Su disponibilidad.

b) Descuentos por volumen.

c) Simplificación de los problemas de almacenamiento.

d) Menores necesidades de capital.

e) Reducción de riesgos en los inventarios.

3. Mejor utilización de las instalaciones de la planta, lo cual tiende a:

a) Reducir la capacidad requerida para cubrir las temporadas pico.

b) Evitar la capacidad ociosa.

\section{Información necesaria}

- Presupuesto de producción en unidades.

- Horas de mano de obra directa por línea de productos.

4 Contaduría Pública de la Nación. Manual de Contabilidad de Costos y de Gestión Industrial. Lima: Dirección General de Contabilidad Analítica de Explotación, 2003, p. 347. 
- Número de trabajadores de planta.

- Tasa media de jornales.

- Prima textil.

- Gratificaciones.

- Compensación por tiempo de servicios.

- Leyes sociales que afectan a las remuneraciones.

FÓRMULA DE PRESUPUESTO DE

MANO DE OBRA DIRECTA

$=$ Unidades de producción requeridas $\mathrm{x}$

Hora de MOD por unidad $\mathrm{x}$ Tasa hora MOD

\section{Presupuesto de los costos indirectos de fabricación}

La mano de obra indirecta se incluye en el presupuesto de costo indirecto de fabricación, es fundamental que la persona encargada del personal lo distribuya de acuerdo a las distintas etapas del proceso de producción para permitir un uso del $100 \%$ de la capacidad de cada trabajador.

Hay dos centros de costos en prácticamente todas las condiciones de costos:

1. Los departamentos productivos.

2. Los departamentos de servicios.

Los primeros son los que trabajan en forma directa en los productos que se elaboran. Los segundos, los que suministran servicios a los departamentos productivos y otros departamentos de servicios.

Los centros de costos de Servicios a la Producción, típicos en una fábrica son:

- Almacenes

- Compras

- Estudios de tiempo y movimiento

- Investigación y desarrollo

- Laboratorios o control de calidad
- Mantenimiento, reparaciones o maestranza

- Planificación y control de la producción

- Seguridad industrial

- Sub-estación eléctrica

Según las bases de actividad que se utilice, las Tasas de Costos Indirectos puede determinarse:

- En función de las unidades de producción.

- En función a las materias primas consumidas.

- En función al uso de la mano de obra directa pagada.

- En función a horas hombre utilizadas.

- En función al costo primo (la suma de los costos directos: materias primas y mano de obra directa).

- En función a las horas máquina.

La departamentalización por centros de costos puede hacerse utilizando dos criterios fundamentales:

1. Patrón Hora - Hombre

2. Patrón Hora - Máquina

Cashin y Polimeni sostienen que los métodos más comunes empleados para asignar los costos de los departamentos de servicios son:

- Método directo

- Método escalonado

- Método algebraico

- Por actividad (costos $A B C)^{5}$

- La combinación de los métodos anteriores.

Método directo. Es el más conocido y el más utilizado dado su simplicidad matemática y su simplicidad de aplicación. Este método hace asignación específica del costo de los departamentos de servicios a los departamentos de producción. De acuerdo con la importancia del servicio prestado e ignora

5 Propuesto por el autor responsable del trabajo de investigación. 
la asignación del costo de servicios a los departamentos de servicios.

Método escalonado. Denominado así porque la distribución de costos del departamento de servicios a los departamentos de producción, se efectúa mediante una serie de pasos realizados en forma escalonada $\mathrm{DE}$ LO PRINCIPAL A LO SECUNDARIO. Es decir, se distribuyen los costos del departamento de servicios que proporcionan servicios al mayor número de los departamentos de servicios. A continuación se distribuye el departamento de servicio que le sigue en importancia. Cualquier costo sumado a este departamento del paso 1 , se incluye. Pero una vez que los costos de una departamento de servicios han sido asignados a otros departamentos, ningún costo de servicios puede serle asignado en el futuro.

Método algebraico. Es el método más preciso de los tres, ya que considera los servicios recíprocos entre secciones en los Departamentos de Servicios. El uso de ecuaciones simultáneas permite la asignación recíproca ya que a cada departamento servido le serán asignados costos de la sección que proporciona el servicio.

Por actividades. Por este método se dividen los costos indirectos en grupos separados de costos de actividades homogéneas. La administración utiliza el criterio de causaefecto para identificar los factores de costos para los grupos separados. Los siguientes son pasos clave para distribuir los servicios con base en actividades:

1. Identificar y definir las actividades relevantes.

2. Organizar las actividades por centros de costos.

3. Identificar los componentes de costos principales.
4. Determinar las relaciones entre actividades y costos.

La combinación de los métodos anteriores. Que no es sino la mezcla de los modelos antes descritos.

\section{Información necesaria}

- Presupuesto de horas de mano de obra directa por centros productivos y servicios.

- Componentes de costos principales.

- Base de distribución de los costos relevantes.

Se dispone de la siguiente información, que es un modelo combinado que distribuye los costos departamentales productivos y de servicios en dos etapas.

1. ${ }^{\text {ra }}$ Etapa. Se reparten los costos en base a tres factores:

- Por área de planta utilizada.

- Por la planilla de remuneraciones pagadas en el periodo.

- Por inversiones en el activo fijo.

\section{Presupuesto costo de artículos vendidos}

El Costo de los artículos fabricados o costo de fabricación es la sumatoria de los siguientes anexos:

- Consumo de materiales directos

- Mano de obra directa

- Costos indirectos de fabricación

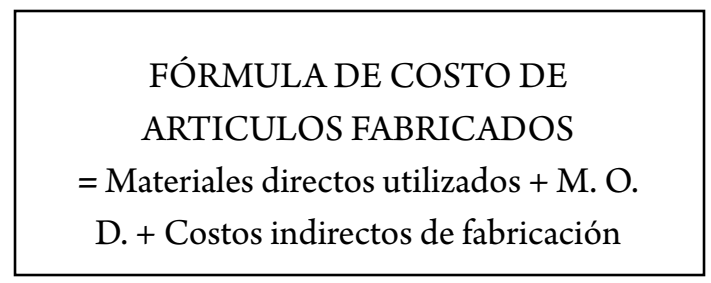

El siguiente paso es calcular el Costo de Artículos Fabricados que está integrado por los siguientes elementos: 
Inventario inicial de productos en proceso

- (+) Costo de Fabricación

- = Fabricación Disponible

- (-) Inventario Final de Productos en Proceso

- = costo de artículos producidos

Un Costo de Fabricación puede convertirse en Costo de Producción, si no existe inventario inicial y final de productos en proceso.

El siguiente paso, a partir del costo de los artículos producidos, procede la preparación del presupuesto del costo de los artículos vendidos. Que es como se muestra en el siguiente cuadro:

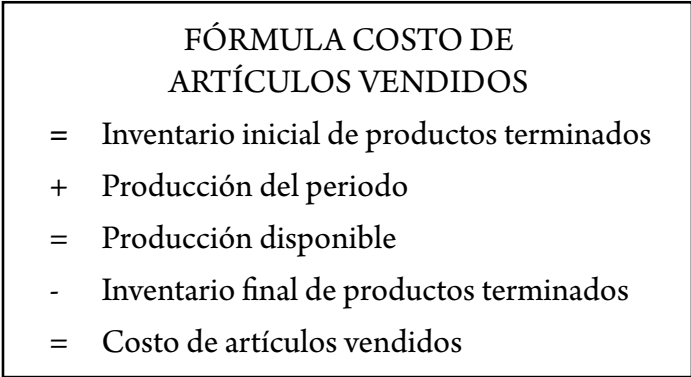

\section{Presupuesto de gasto de ventas}

Es el Presupuesto de mayor cuidado en su manejo por los gastos que ocasiona y su influencia en el gasto financiero. Comprende todos los gastos relacionados con la actividad de vender, la distribución y la entrega de los productos a los clientes durante todo el proceso de comercialización para asegurar la colocación y adquisición del mismo en los mercados de consumo. La cuidadosa planificación de dichos gastos afecta favorablemente al potencial de utilidades de la empresa.

Para determinar la asignación promocional y de publicidad se emplean varios métodos, y los más comunes son: a) Asignación arbitraria, b) Totalidad de fondos disponibles, c) Igualdad competitiva, d) Un porcen- taje de las ventas, e) Una suma fija por unidad vendida, f) Utilidades del año pasado, g) Rendimiento sobre la inversión y h) El método de tareas específicas.

\section{Información necesaria}

- Presupuesto de ventas en valores monetarios.

- Gastos variables.

- Gastos fijos.

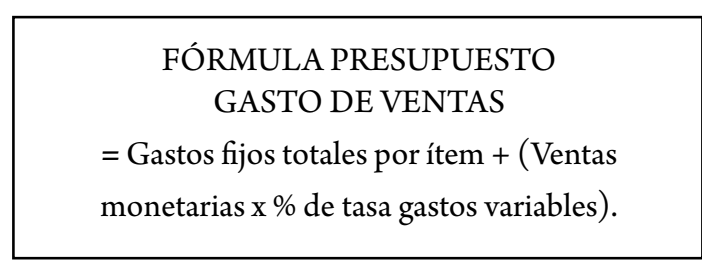

\section{Presupuesto de gastos administrativos}

Son gastos de administración todos los gastos que no sean de producción ni de distribución buscando darle operatividad al sistema. Debe ser lo más austero posible sin que ello implique un retraso en el manejo de los planes y programas de la empresa. Se incluyen el pago de salarios a la Gerencia General y al personal Staff.

La administración central en cualquier compañía, excepto en las muy pequeñas, se ejerce en varias áreas de responsabilidad especiales, como son gerencia general, finanzas, tesorería, personal, y el grupo central de asesoría. Por consiguiente, este presupuesto abarca varios presupuestos departamentales.

\section{Información necesaria}

- Gastos fijos.

$$
\begin{aligned}
& \text { FÓRMULA PRESUPUESTO } \\
& \text { GASTOS DE ADMINISTRACIÓN } \\
& \text { = Suma de los gastos fijos }
\end{aligned}
$$




\section{Presupuesto de gastos financieros}

Los gastos financieros en su mayor parte dependen del costo de fabricación por lo que, normalmente, su incidencia suele calcularse aplicando un porcentaje a dicho costo excluída la amortización. Sin embargo, resulta más lógico calcularlos según la incidencia de los diversos componentes del costo. De esta manera, el costo financiero ha de variar de acuerdo a la cantidad de meses de acopio de materia prima y/o de productos terminados, del tiempo de fabricación, como también del interés que paga el cliente por un lado y del plazo sin interés que nos otorga el proveedor, por otro lado 6 .

Gastos financieros: Estos se relacionan con la obtención de fondos para la operación de la empresa. Incluyen el costo de los intereses que la compañía debe pagar por los préstamos, así como el costo de otorgar crédito a clientes.

Considerando que los gastos financieros dependen en forma proporcional del grado de actividad de la empresa, en general tienen la calidad de "gastos variables". Tienen carácter de gastos "fijos" aquellos que surgen de costos fijos (Producción, administración y ventas). Muchas veces surge la discusión respecto de la carga del interés cuando la compañía dispone de suficiente capital operativo propio y prescinde parcial o totalmente de créditos externos. Nuestro criterio es que en una economía sana, siempre deberá calcularse un interés aunque contablemente se origine una ganancia. El sustento está en que una empresa no tendría necesidad de funcionar si puede obtener una renta de su capital depositándolo en una cuenta de ahorros.

\section{Estado de ganancias y pérdidas proyectado}

El resultado final del Presupuesto de Operaciones, es la formulación del estado Proforma de Ganancias y Pérdidas, reuniendo y combinando las cifras presupuestales pertinentes ya desarrolladas como se muestran a continuación:

Anexo N. ${ }^{\circ} 12$. Ganancias y pérdidas mediante costeo por absorción

Anexo N. ${ }^{\circ}$ 12.1. Ganancias y pérdidas mediante costeo directo.

Anexo N.o 12.2. Ganancias y pérdidas por línea de productos mediante costeo por absorción

Anexo N.o 12.3. Ganancias y pérdidas por línea de productos mediante costeo directo.

\section{El presupuesto de inversiones}

Para Glenn Welsch", "un desembolso de capital es el uso de fondos, esto es, de efectivo, para adquirir activos operacionales que: a) ayuden a generar futuros ingresos o b) reduzcan futuros costos....”

De esta manera se plantean necesidades de distinto tipo, para:

a) Nuevas líneas de productos.

b) Reemplazos por obsolescencia.

c) Mejorar el nivel de costos

d) Mejorar el nivel de producción

e) Instalación de oficinas (acondicionadores de aire, amoblamiento, equipos de computación de datos, máquinas de oficina, vehículos para vendedores, etc.).

f) Investigación aplicada común en las industrias químicas y electrónicas.

6 Bidart, Jorge Adolfo. "El Presupuesto en la Empresa. Metodología de un caso práctico". Editorial Macchi. Buenos Aires. Argentina. Edición 2000. Páginas 78-84.

7 Welsch, Glenn. Hilton, Ronald W. Gordon, Paul N. “Presupuesto. Planificación y Control de Utilidades”. Editorial Prentice Hall Hispanoamérica S.A. México. Quinta Edición 1990. Página 419. 
g) La formación, perfeccionamiento y capacitación del personal.

h) Inversión comercial para dar a conocer una marca o para lanzar un nuevo producto.

\section{Los criterios económicos para financiar los desembolsos de capital}

Existen tres conceptos de financiamiento de las inversiones: el "costo de capital", el "costo de oportunidad" y el "costo ponderado del capital".

1. El costo de capital está relacionado con los fondos tomados en préstamo en base a capital ajeno. Si acudimos a un Banco el costo de capital es la tasa que exigirá el Banco por otorgar el préstamo. Supongamos $25 \%$ anual TAMN (Tasa anual en moneda nacional).

2. El costo de oportunidad se sustenta en los fondos propios relacionado a "los rendimientos que se dejan de percibir por no emplear dichos fondos, en otros proyectos posibles, en iguales condiciones de riesgo". El costo de oportunidad es la utilidad que el inversionista desea recibir por invertir en el proyecto. Generalmente este costo es superior al costo del préstamo bancario. Supongamos $35 \%$.

3. El costo ponderado del capital es el promedio ponderado de la participación del capital ajeno y el capital propio en el financiamiento del proyecto. Si cada inversionista (capital ajeno y propio), participara en partes iguales en el financiamiento de proyecto, el costo ponderado sería $(25+35) / 2=30 \%$. La decisión de elegir las fuentes de financiamiento dependen en la realidad, del monto del proyecto, de la rentabilidad proyecta $y / o$ de la disponibilidad de los fondos.

\section{Métodos de medición del valor económico de un desembolso de capital}

Existen numerosos métodos de medición del valor económico de una inversión, pero los de uso más generalizado son:

1. Métodos del flujo de efectivo descontado

a) El del valor actual neto (VAN).

b) El de la tasa interna de rendimiento o de retorno (TIR).

2. Métodos abreviados y simples

a) El del periodo de recuperación.

b) El de la tasa contable de rendimiento.

Los métodos basados en el flujo de efectivo descontado, reconocen explícitamente los efectos del valor del dinero en el tiempo. Que consiste en actualizar todos los flujos monetarios percibidos o generados por la inversión a una fecha dada. De todas las técnicas usadas en finanzas ninguna es más importante que el valor del dinero en el tiempo.

El valor actual, son los flujos descontados a una tasa de rentabilidad.

$$
\mathrm{VA}=\frac{\mathrm{C}_{1}}{(1+\mathrm{i})}
$$

El valor actual neto, o valor presente neto, es el valor actual de los flujos menos la inversión inicial.

VAN = VA - INVERSIÓN REQUERIDA

FÓRMULA GENERAL
VAN $=-\mathrm{C}_{0}+\frac{\mathrm{C}_{1}}{(1+\mathrm{i})}+\frac{\mathrm{C}_{2}}{(1+\mathrm{i})^{2}}+\frac{\mathrm{C}_{3}}{(1+\mathrm{i})^{3}}+\ldots \ldots \ldots \frac{\mathrm{C}^{\mathrm{n}}}{(1+\mathrm{i})^{\mathrm{n}}}$

Donde:

$\mathrm{C}_{0}=$ Inversión inicial

$\mathrm{C}_{1} \quad=$ Flujo de tesorería

$(1+\mathrm{i})=$ Tasa de descuento o costo de capital o costo de oportunidad.

n $\quad=$ Vida útil del proyecto. 
Criterios de Decisiones en función del Valor Actual Neto.

\begin{tabular}{|c|c|}
\hline CONCEPTOS & RESULTADO \\
\hline Si VAN $=0$ & Indiferente \\
\hline Si VAN $>0$ & Aceptar el proyecto \\
\hline Si VAN $<0$ & Descartar el proyecto \\
\hline
\end{tabular}

Se aceptan siempre las inversiones que tienen un valor actual neto superior.

\section{Criterios de decisión en función del índice de rentabilidad}

Aceptar las inversiones que ofrecen tasas de rentabilidad que superen el costo de oportunidad del capital.

Supongamos el siguiente ejemplo:

La Consultora "Beta SAC" se encuentra analizando la posibilidad de llevar a cabo uno de tres proyectos de inversión en el área de electrificación rural con fines industriales para empresas dedicadas a sustitución de importaciones, para tal efecto se ha reunido la siguiente información relativa a los Proyectos en mención, después de un periodo presupuestado de análisis futuro de 5 años de horizonte.

\begin{tabular}{|l|c|c|c|}
\hline \multirow{2}{*}{$\begin{array}{c}\text { CONDICIONES } \\
\text { ECONÓMICAS } \\
\text { DEL VAN }\end{array}$} & \multicolumn{3}{|c|}{$\begin{array}{c}\text { VALOR ACTUAL NETO EN EL AÑ } \\
\text { “0” (EN MILES DE DÓLARES) }\end{array}$} \\
\cline { 2 - 4 } & $\begin{array}{c}\text { Proyecto } \\
\text { "A" }\end{array}$ & $\begin{array}{c}\text { Proyecto } \\
\text { "B” }\end{array}$ & $\begin{array}{c}\text { Proyecto } \\
\text { "C” }\end{array}$ \\
\hline Muy malas & $-4,000$ & 800 & -400 \\
\hline Malas & $-1,000$ & 1,000 & 100 \\
\hline Regulares & 3,500 & 2,500 & 1,000 \\
\hline Buenas & 4,000 & 3,000 & 2,000 \\
\hline Muy buenas & 6,500 & 3,700 & 3,200 \\
\hline
\end{tabular}

Además se conoce que las condiciones económicas y la probabilidad de ocurrencia de los VAN esperados para cada Proyecto, tienen la siguiente relación:

\begin{tabular}{|c|c|c|c|c|c|}
\hline $\begin{array}{c}\text { CONDICIONES } \\
\text { ECONÓMICAS }\end{array}$ & $\begin{array}{c}\text { MUY } \\
\text { MALAS }\end{array}$ & MALAS & REGULARES & BUENAS & $\begin{array}{c}\text { MUY } \\
\text { BUENAS }\end{array}$ \\
\hline $\begin{array}{c}\text { PROBABILIDAD } \\
\text { OCURRENCIA } \\
\text { DEL VAN }\end{array}$ & 0.1 & 0.2 & 0.4 & 0.2 & 0.1 \\
\hline
\end{tabular}

Si el monto de la inversión total para cualquiera de los tres proyectos es de US\$ 1'125,000 y además se sabe que la elección del mejor proyecto de inversión se hará en función del mejor índice B/C (Beneficio/ Costo), se solicita determinar lo siguiente:

1. Determine el valor esperado del VAN de los proyectos en mención.

2. Determine el proyecto seleccionado y,

3. Justifique técnicamente su decisión.

\section{Tasa interna de retorno o flujo de tesorería descontado}

Es aquel tipo de actualización que hace igual a cero el valor actual neto.

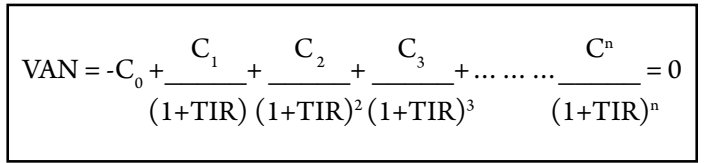

Hallar la TIR del Proyecto "A". Costo de Oportunidad $10 \%$.

\begin{tabular}{|c|c|c|}
\hline PROYECTO & INVERSIÓN & $\begin{array}{c}\text { FLUJO DE } \\
\text { FONDOS }\end{array}$ \\
\hline \multirow{2}{*}{ "A" } & -200 & 1234 \\
\cline { 3 - 3 } & & 70706060 \\
\hline
\end{tabular}

Resultado: En el presente caso la TIR es $11.79 \%$. Superior al 10\% del Costo de Oportunidad. Por tanto se acepta el proyecto.

\section{Criterio de selección en función de la tasa interna de retorno}

En situaciones normales se acepta el proyecto si la tasa supera el costo de oportunidad del Capital. Si existe discrepancia con el VAN, se acepta el criterio del VAN. 


\section{El periodo de recuperación}

Regla. Cuando los flujos de fondos no son constantes, el periodo de recuperación se determinará acumulando lo sucesivo a flujos anuales. Ejemplo:

\begin{tabular}{|c|c|c|}
\hline \multirow{2}{*}{ PROYECTO } & \multirow{2}{*}{ INVERSIÓN } & $\begin{array}{c}\text { FLUJO DE } \\
\text { FONDOS }\end{array}$ \\
\cline { 3 - 3 } & & 1234 \\
\hline $\mathrm{A}$ & 200 & 70706060 \\
\hline $\mathrm{B}$ & 170 & 60504555 \\
\hline $\mathrm{C}$ & 120 & 5050500 \\
\hline
\end{tabular}

\section{Criterio de decisión}

Seleccionar el proyecto de inversión que se recupera en un periodo de tiempo más corto. Da la misma ponderación a todos los flujos de tesorería generados antes de la fecha correspondiente al periodo de recuperación y una ponderación nula a todos los flujos posteriores. La Empresa tiene que decidir una fecha tope adecuada, independiente de la vida del proyecto.

\section{Resultados}

\begin{tabular}{|c|c|}
\hline PROYECTOS & $\begin{array}{c}\text { PLAZO DE } \\
\text { RECUPERACIÓN }\end{array}$ \\
\hline A & 3.3 años \\
\hline B & 3.25 años \\
\hline C & 2.40 años \\
\hline $\begin{array}{c}\text { Se elige el proyectro C porque se recupera en un } \\
\text { periodo de tiempo más corto. }\end{array}$ \\
\hline
\end{tabular}

\section{Tasa contable de rendimiento o beneficio costo}

Consiste en establecer una relación entre los resultados derivados de la inversión (flujo de caja anual), y el costo de las mismas.

\section{Criterio de decisión}

La mejor inversión será aquella que presente una relación beneficio-costo superior. Supongamos el mismo caso anterior:

\begin{tabular}{|c|c|c|}
\hline \multirow{2}{*}{ PROYECTO } & INVERSIÓN & $\begin{array}{c}\text { FLUJO DE } \\
\text { FONDOS }\end{array}$ \\
\cline { 3 - 3 } & & 1234 \\
\hline $\mathrm{A}$ & 200 & 70706060 \\
\hline $\mathrm{B}$ & 170 & 60504555 \\
\hline $\mathrm{C}$ & 120 & 5050500 \\
\hline
\end{tabular}

\section{Resultado}

\begin{tabular}{|c|c|c|}
\hline A & $260 / 200$ & $30.00 \%$ \\
\hline B & $210 / 170$ & $23.53 \%$ \\
\hline C & $150 / 120$ & $25.00 \%$ \\
\hline \multicolumn{3}{|c|}{ Se elige el proyecto A porque su rentabilidad es } \\
la mayor. \\
\hline
\end{tabular}

\section{El presupuesto financiero}

Luego de elaborado los diversos presupuestos básicos ya podemos pasar a la etapa final., o sea el Presupuesto Financiero que incluye las provisiones de tesorería (ingresos y egresos que derivan de la actividad planeada.

En primer lugar es necesario establecer si es posible "financiar" la actividad planeada, con el capital operativo disponible, ya que en caso contrario habrá que revisarla y producir las modificaciones para adecuarla conforme a los medios de pago que se dispondrían (incluido el crédito). Estas modificaciones pueden consistir en reducción o cambios en la composición de las ventas, disminución stocks, postergación o suspensión de inversiones u otras medidas apropiadas. De esta manera todo el planeamiento presupuestario anterior queda subordinado al resultado financiero ${ }^{8}$.

8 Bidart, José Adolfo. El Presupuesto en la Empresa. Metodología de un Caso Práctico. Buenos Aires: Ediciones Macchi, p. 151. 
Concretamente el problema se plantea en establecer, durante el periodo presupuestario, si el flujo de fondos esperado permite atender en cantidad y oportunidad los pagos de la empresa, conforme a los compromisos $\mathrm{u}$ obligaciones que implica la actividad planeada.

\section{El flujo de caja}

Denominado también Presupuesto de Caja, Presupuesto de Tesorería o Cash Flow, es un instrumento que permite mantener un adecuado control sobre las disponibilidades del efectivo y/o la liquidez en determinados periodos. Es de gran ayuda en el manejo financiero empresarial. Para una empresa es útil tener información oportuna acerca del comportamiento de su caja permitiendo una administración óptima.

Consiste en fijar los estimados de venta, ingresos varios para elaborar al final un flujo de caja que mida el estado económico y real de la empresa, comprende:

- Presupuesto de ingresos (el total bruto sin descontar gastos)

- Presupuesto de egresos (para determinar el líquido o neto)

- Flujo neto (diferencia entre ingreso y egreso)

- Caja final

- Caja inicial

- Caja mínima

\section{Procedimientos a seguir para elaborar un} flujo de caja

1. Elaborar el presupuesto de ventas

2. Elaborar el presupuesto de cobranzas

3. Elaborar el presupuesto de compras

4. Elaborar el presupuesto de gastos de ventas
5. Elaborar el presupuesto de gastos de administración.

6. Elaborar el presupuesto de gastos financieros.

7. Finalmente, el flujo de caja, resume todos los presupuestos anteriores.

Por ello, en nuestro trabajo exponemos un método general que será preciso adecuar a cada caso particular.

\section{Modelo de pronóstico de caja con financiamiento}

\begin{tabular}{|l|l|l|l|l|l|}
\hline \multicolumn{1}{|c|}{ CONCEPTOS } & ENE & FEB & $\ldots \ldots .$. & DIC & TOTAL \\
\hline 1. Entradas de efectivo & & & $\ldots \ldots .$. & & \\
\hline 2. Salidas de efectivo & & & $\ldots \ldots .$. & & \\
\hline 3. Flujo neto de caja & & & $\ldots \ldots .$. & & \\
\hline 4. Saldo inicial de caja & & & $\ldots \ldots .$. & & \\
\hline 5. Saldo final de caja & & & $\ldots \ldots .$. & & \\
\hline $\begin{array}{l}\text { 6. Financiamiento } \\
\text { 7. Saldo de caja con } \\
\text { financiamiento }\end{array}$ & & & $\ldots \ldots .$. & & \\
\hline
\end{tabular}

Modelo de presupuesto de caja

\begin{tabular}{|l|l|l|l|l|l|}
\hline \multicolumn{1}{|c|}{ CONCEPTOS } & ENE & FEB & $\ldots \ldots .$. & DIC & TOTAL \\
\hline Saldo inicial & & & $\ldots \ldots .$. & & \\
\hline 1. Entradas en efectivo & & & $\ldots \ldots .$. & & \\
\hline TOTAL INGRESOS & & & $\ldots \ldots .$. & & \\
\hline 2. Salidas de efectivo & & & $\ldots \ldots .$. & & \\
\hline SUB-TOTAL & & & $\ldots \ldots .$. & & \\
\hline 3. Saldo final de caja & & & $\ldots \ldots .$. & & \\
\hline TOTAL EGRESOS & & & $\ldots \ldots .$. & & \\
\hline
\end{tabular}

\section{El análisis del punto de equilibrio}

Denominado también "Análisis de Costo Volumen-Utilidad y de Contribución”, implica el empleo de una serie de técnicas ana- 
líticas para determinar y evaluar los efectos sobre las utilidades, de: los cambios en el volumen de ventas, es decir, en las unidades vendidas, en los precios de venta, en los costos fijos y en los costos variables. Se enfoca en el margen de contribución, que es el ingreso por ventas menos los costos variables totales, igual al margen de contribución; y margen de contribución menos costos fijos, igual a la utilidad. El análisis de equilibrio se enfoca sobre el punto de equilibrio: gastos fijos divididos entre el margen de contribución, igual al volumen de ventas de equilibrio (el punto en el cual la utilidad es cero, porque los ingresos son iguales al costo total.

Un estado de resultados presupuestado bajo el enfoque del margen de contribución, permite responder a numerosas preguntas de "qué pasa si...":

1. ¿El volumen de utilidades disminuyera en un $5 \%$ ?

2. ¿Cuál sería la utilidad si el precio de venta se incrementara en un 5\%?

3. ¿Cuál sería la utilidad si los costos fijos aumentaran en un $10 \%$ ?

4. ¿Cuál sería la utilidad si los costos variables se redujeran en un 5\%?

5. ¿Cuál sería la utilidad si tuvieran lugar los cuatros cambios que se indican en los casos anteriores?

\section{El balance general proyectado}

Cuando el análisis de la información planificada ha llegado a este nivel, la aprobación del presupuesto definitivo implica la obtención de una respuesta afirmativa a los interrogantes:

1. ¿La capacidad de producción permite atender las ventas planeadas?

2. ¿Satisfacen las inversiones las necesidades de la producción?
3. ¿Los costos y gastos permiten vender con ganancia?

4. ¿Son suficentes y están bien aplicados los recursos financieros?

5. ¿Hay suficiente liquidez para atender al normal funcionamiento de las actividades?

6. ¿Se han cumplido con las metas cuantificadas propuestas por la Gerencia?

Una vez terminado los balances proyectados mensuales, se puede hacer el análisis de la evolución que acusaría la empresa en su situación económico-financiera y patrimonial a través de los divesos índices que conocemos tales como: liquidez, gestión, endeudamiento y rentabilidad.

\section{MODELO A UTILIZARSE EN LA PRESENTACIÓN DEL PLAN ANUAL DE UTILIDADES}

Si en el Perú se ordenara el uso obligatorio del planeamiento empresarial, en base al modelo bajo análisis o cualesquier a otro que pudiera sugerirse, las situaciones de coyuntura podrían fácilmente encontrar alternativas de solución. Se propone el siguiente formato de Plan Anual y Aplicación de Utilidades para la empresa industrial, teniendo en cuenta los siguientes principios de contabilidad generalmente aceptado:

1. El costo a valor razonable.

2. Uniformidad o Consistencia, elegido una forma de calcular los costos, no es posible modificarlos en el transcurso del ejercicio vigente.

3. Periodo contable, que las operaciones se realicen de enero a diciembre de cualquier año corriente.

A continuación el formato presentado en todo el trabajo de investigación:

- El pronóstico de ventas y el presupuesto de ventas consolidado. 
- El presupuesto de compras de materiales y del de consumo de materiales.

- El presupuesto de mano de obra directa.

- El presupuesto de carga fabril o gastos indirectos de fabricación.

- El presupuesto de costo de producción.

- El presupuesto de costo de ventas y gasto de ventas.
- El presupuesto de gastos de administración y gastos financieros.

- El presupuesto de adquisición de activos fijos.

- El flujo de caja proyectado, así como estado de ganancias y pérdidas proyectado.

- El cálculo del punto de equilibrio por línea de productos.

- El balance general proyectado.

\section{EL PLAN ANUAL DE UTILIDADES. SU DESARROLLO Y COMPOSICIÓN}

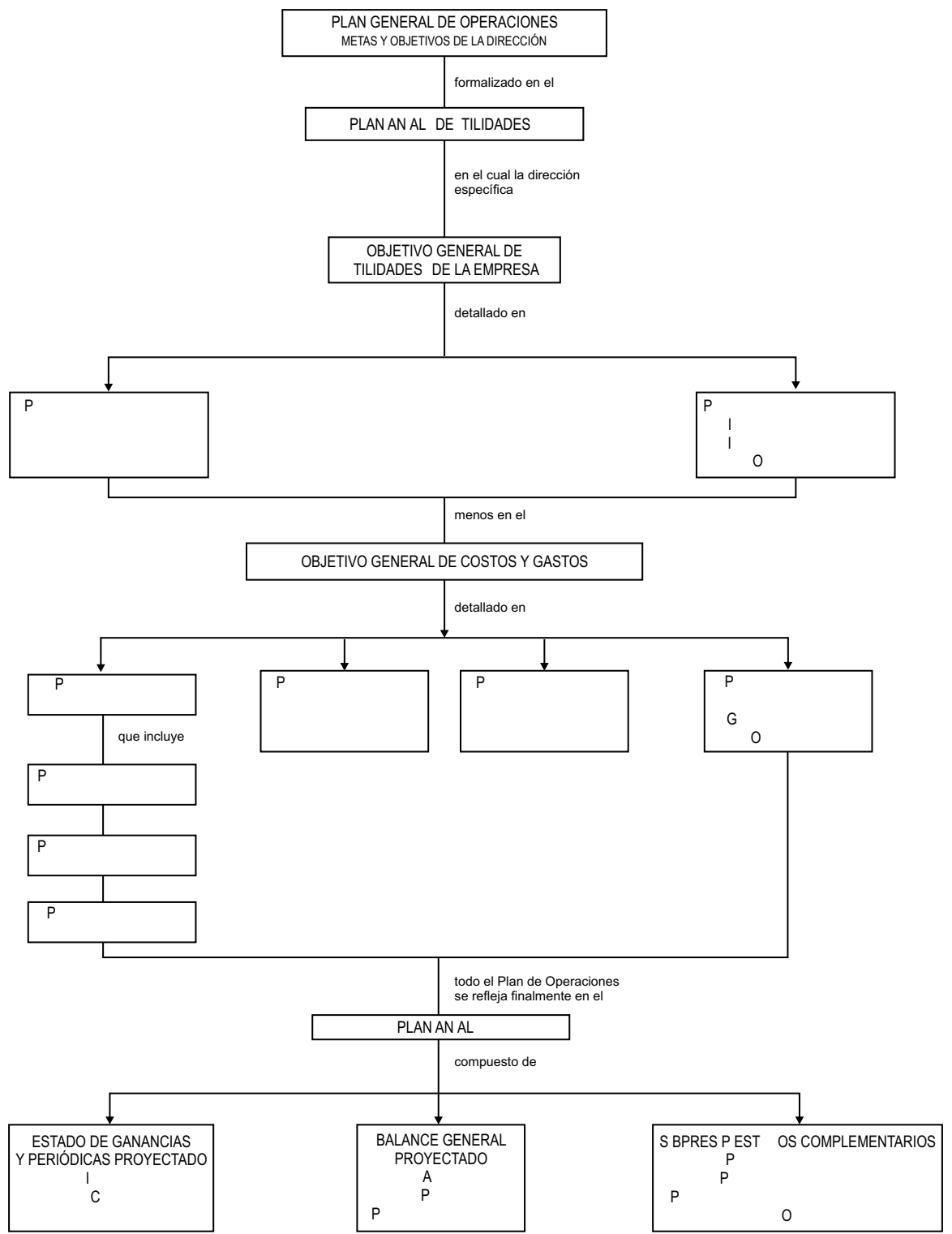

84/ QUIPURAMAYoc | Vol. 17(2) 2010 


\section{CONCLUSIONES}

Una consideración básica y fundamental que no se discute en el ámbito empresarial, radica en la posibilidad de controlar la previsión presupuestaria. Es por ello que resulta necesario establecer, paralelamente con el planeamiento, el sistema de control presupuestario adecuado, que consiste en la confrontación de lo previsto con lo realizado.

1. Como la operación de todas las dependencias de la compañía ha sido presupuestada, las actividades de cada departamento o sección deben estar bajo el control presupuestal con el doble objetivo de vigilar la marcha prevista y adoptar los correctivos ante las desviaciones significativas que se observasen.

2. Las decisiones de negocios primero se planifican: materias primas, mano de obra, carga fabril, volumen de producción más conveniente, márgenes de utilidad, costo de capital, etc. Luego se decide atender o no un pedido, por tanto, ser competitivos en el mundo actual, es tomar la decisión correcta y la práctica de estas técnicas impulsan a la administración a planificar el empleo más económico de los factores de la produccion. La facturación y los registros contables nos proporcionan la información cierta de las ventas realizadas. La simple comparación de los valores monetarios previstos con la meta de las ventas realizadas, puede llegar a mostrar un cuadro más favorable cuando se han operado aumentos de precios.

3. En un mundo donde las cosas cambian aceleradamente, esta herramienta resulta fundamental en nuestros países que no tienen el concepto de prever antes que solucionar. Desarrollar una cultura prospectiva en el recurso humano empresarial medio, como son las MYPES, es un crecimiento cualitativo porque crea el hábito de la consideración oportuna, cuidadosa y apropiada de todos los factores pertinentes, antes de tomar decisiones trascendentes.

\section{RECOMENDACIONES}

1. La Ley 24680 prevé en su título III, Art. $7^{\circ}$, inc. b, que es función de Contaduría Pública de la Nación, planear, organizar, y coordinar el estudio e investigación de las actividades económicas, de los organismos del sector público y privado. Los Institutos de Investigación del Sector Económico-Empresarial de nuestra Universidad, deberían promover alianzas estratégicas de estudio con esta entidad, contribuyendo a la realización de sus fines y a la promoción del planeamiento de utilidades en la MYPES peruanas.

2. El dominio de las técnicas de costos íntimamente ligadas a la planificación de utilidades, se recomienda a nuestra Facultad de Ciencias Contables, incluir en su nueva Escuela Académico-Profesional de Contabilidad Planes Curriculares que incluyan cursos permanentes de Costos Industriales, Costo-Volumen-Utilidad, Toma de Decisiones, Costo Basado en Actividades, Contabilidad por Áreas de Responsabilidad, Costeo de Inventarios y Análisis de Rentabilidad, Decisiones de Fijación de Precios, Presupuestación de Capital y Análisis Costos, etc., materias fundamentales para preparar especialistas en Sistemas de Planeación y Control de Utilidades tanto para las MYPES y para las distintas ramas de la actividad económica del país, lo que ayudará a mejorar la toma de decisiones y el uso adecuado de los escasos recursos de estas empresas. 
3. Nuestras instituciones tutelares se preocupan, como es justo, de legislar el registro y la presentación uniforme de la información contable pasada que es importante, pero no existe normalización alguna sobre el planeamiento y control prospectivo de las actividades. Lo que permitiría definir por adelantado que lleven al mejoramiento en la productividad del flujo de fondos, el uso alternativo de la capacidad ociosa, la calidad, la gestión y la competitividad en todos los sectores de la economía nacional, lo que facilitaría la tarea de exportación en que se encuentra inmerso la industria nacional para superar los problemas de falta de puestos de trabajo. Donde la colaboración del Colegio de Contadores Públicos del Perú, resulta indispensable para promover su difusión.

\section{REFERENCIAS BIBLIOGRÁFICAS}

1. Antony, Robert; Govindaraján, Vijay. "Sistemas de Control de Gestión”. Décima Edición. Madrid: Editorial McGrawHill / Interamericana de España, 2003.
2. Apaza Meza, Mario. "Costos $A B C, A B M$, ABB. Herramientas para Incrementar la Rentabilidad y la Competitividad Empresarial”. Lima: Editoria y Distribuidora Real SRL, 2003.

3. Hicks, Douglas. "El Sistema de Costos basado en las actividades ( $\mathrm{ABC}$ ). Guía para su implantación en pequeñas y medianas empresas. México: Editorial Alfaomega, 1998.

4. Horngren, Charles T.; Foster, George; Datar, Srikant. "Contabilidad de Costos. Un Enfoque Gerencial”. México: Editorial Prentice-Hall Hispanoamericana SA., 1996.

5. Horngren, Charles T. Sundem, Gary L. Selto, Frank. "Contabilidad Administrativa”. Novena edición. México: Editorial Prentice-Hall Hispanoamericana S.A., 1993.

6. Polimeni, Ralph; Fabozzi, Frank; Adelberg, Arthur. "Contabilidad de Costos. Conceptos y Aplicaciones para la toma de decisones gerenciales". Tercera edición. Bogotá: Editorial McGraw-Hill, 1994.

7. Welsch, Glenn; Hilton, Ronald; W. Gordon; Paul N. "Presupuestos. Planificación y Control de Utilidades". Quinta Edición. México, 1990. 